\title{
Konsequenzen des zweiten demographischen Übergangs für den ländlichen Raum Alternative Konzepte zur Nahversorgung aufgezeigt an der Region Nordhessen
}

\section{Florian Warburg, Marburg}

\section{Einleitung}

Der zweite demographische Übergang - oft aber fälschlicherweise mit dem Begriff demographischer Wandel gleichgesetzt - ist vorrangig eine in Industrienationen auftretende Entwicklung der Abnahme und Alterung der postindustriellen Gesellschaften und in den meisten europäischen Staaten allgegenwärtig (KERTZER et al. 2009; Lesthaeghe \& NeIdert 2006; Siedhoff 2008) Ländliche Räume sind in besonderem Masse von eben diesem betroffen, da die hohe Abwanderung zum generellen Geburtenrückgang additiv wirkt (PINILLA et al. 2008; WALFORD \& KuREK 2008). Begründet liegt der demographische Transformationsprozess in einer seit Beginn der 1970er Jahre stattfindenden Veränderung von Werten und Normen. Diese auch als Wertewandel zusammenzufassenden Entwicklungen finden u.a. Ausdruck in einem veränderten Konsumverhalten der unterschiedlichen Bevölkerungsgruppen bzw. Gesellschaftsschichten (KuHLICKE \& PeTschow 2005; PurPer 2007). Aufgrund dieser Vorgänge verändert sich die Nahversorgungslage, das heisst die Nachfrage nach Waren und Dienstleistungen des täglichen Bedarfes, aber auch die Anforderungen an technische und soziale Infrastruktur in ruralen Siedlungen. Dies birgt Probleme für die Bewohner der ländlichen Räume, gerade auch für ältere und körperlich eingeschränkte Menschen, die in ihrer Mobilität und damit in der eigenständigen Versorgung beeinträchtigt sind (CIRKEL \& JuCHELKA 2007). An dieser Stelle sei zu erwähnen, dass es nicht «den ländlichen Raum», sondern vielmehr ein breites Spektrum an Typisierungen ländlicher Räume, und keinesfalls eine einfache Stadt-Land-Dichotomie gibt (Born 2011). Nicht nur das Konsumverhalten ist einer ständigen Veränderung unterworfen. Parallel zu den Entwicklungen der Bevölkerungszahl und -zusammensetzung gibt es verschiedene Trends auf der Angebotsseite, die in hohem Ausmass die ländlichen Regionen affizieren. Die voranschreitende Konsolidierung im Lebensmitteleinzelhandel, einhergehend mit einem Konzentrationsprozess der Versorgungseinrichtungen, führt zu einer «Ausdünnung des Versorgungsnetzes» sowie einer «funktionalen Erosion nicht zentraler ländlicher Ortschaften» (METzGER 2008: 10).

Im Bereich der Nahversorgungsthematik gibt es bereits eine Reihe von Untersuchungen. In einer Studie über den Raum Stuttgart, welche provokativ im
Titel die Frage aufwirft, ob «Das Ende der Nahversorgung?» bereits eingeleitet wurde, wird der Fokus der Untersuchung zwar auf die Entwicklungen im Einzelhandel sowie auf den Einfluss der Raumordnung gelegt, jedoch kommt der ländliche Raum etwas $\mathrm{zu}$ kurz (Verband Region Stuttgart 2001). KuHLicke et al. (2005) stellen in der Studie «Versorgung mit Waren des täglichen Bedarfs im ländlichen Raum» die Veränderungen auf der Angebots- und Nachfrageseite dar und zeigen alternative Versorgungsmöglichkeiten auf. Auch neueste Untersuchungen, welche die Auswirkungen des demographischen Transformationsprozesses auf andere Bereiche thematisieren, zeigen die grosse Bedeutung dieses Forschungsfeldes (HilligardT 2010; KüPPER 2010; MATuschewsKi 2010; Neu 2011).

Ziel dieses Beitrags ist es, exemplarisch die Konsequenzen des zweiten demographischen Übergangs auf die Nahversorgung in ländlichen Räumen darzustellen und Alternativen aufzuzeigen. Die Zusammenhänge werden auf theoretischer Betrachtungsebene in Abschnitt 2 dargestellt. Dabei wird die Frage aufgeworfen, inwiefern in diesem Kontext Lücken in der Versorgungssituation entstehen und wie diese geschlossen werden können. Untersuchungsraum ist die Region Nordhessen, in der die Datenerhebung Ende 2009 bis Ende 2010 mittels fünfundzwanzig leitfadengestützter Experteninterviews durchgeführt wurde. Bei der Erhebung wurde der Fokus auf die Akteure aus der Kommunal- und Regionalpolitik sowie-administration und auf die regional agierenden Institutionen der Bereiche Förderung, Beratung und Ehrenamt gelegt, die als Experten Kenntnisse über die Region und Kommunen einerseits und über ein spezielles Kontextwissen andererseits verfügen. Daneben wurden privatwirtschaftlich agierende und auf Basis ehrenamtlicher Tätigkeit beruhende Nahversorger aus dem lokalen Lebensmitteleinzelhandel und dem lebensmittelverarbeitenden Handwerk befragt. Mithilfe der qualitativen Inhaltsanalyse (MAYRING 2002) wurden die Experteninterviews ausgewertet und die Ergebnisse in Abschnitt 3 im Zusammenhang mit einer regionalen Betrachtung der Bevölkerungs- und Einzelhandelsentwicklung zusammengefasst.

\section{Der demographische Transformationsprozess und seine Auswirkungen auf die Nachfrage}

Der zweite demographische Übergang ist aufgrund des allgemeinen Geburtenrückgangs und der Alte- 
rung der Bevölkerung sowie aufgrund der tendenziell höheren Abwanderung aus ländlichen Regionen Auslöser für die Veränderung von Bevölkerungszahl und -zusammensetzung. Aufgrund von Individualisierungstendenzen der jüngeren Bevölkerungsgruppen ergeben sich neue Formen des Zusammenlebens, die nach Lesthaeghe \& NeIDERT (2006) nicht nur auf veränderte sozio-ökonomische Bedingungen, sondern auf Bedürfnisse höherer Ordnung zurückzuführen sind, z.B. Selbstverwirklichung oder das Bedürfnis nach Anerkennung. Zudem wird der zweite demographische Übergang durch eine bedeutende Verschiebung des Erstheiratsalters nach hinten sowie eine Erhöhung des Anteils der Geburten von unverheirateten Paaren charakterisiert. Dies liegt u.a. in der Verschiebung vom Familismus zur Selbstrealisation und in einer zunehmenden Säkularisierung begründet (KERTZER et al. 2009). Diese Entwicklungen in Kombination mit einem Absinken der Geburtenrate unter das Reproduktionsniveau sowie einer zunehmenden Alterung der Bevölkerung führen zwar zu einem Anstieg der absoluten Anzahl an Haushalten, jedoch gleichzeitig zur Abnahme der Haushaltsgrösse. Schliesslich sinkt die Bevölkerungszahl stetig und die natürliche Bevölkerungsabnahme kann auch durch die Zuwanderung nicht mehr ausgeglichen werden. Dabei sind die Aussen- und Binnenwanderungen keine direkten Aspekte des zweiten demographischen Übergangs, werden jedoch im Allgemeinen als charakteristisches Merkmal angesehen (SIEDHOFF 2008).

Aufgrund der Individualisierungstendenzen und der Verkleinerung der Haushalte in Kombination mit der Alterung der Gesellschaft nimmt die Bedeutung von helfenden Tätigkeiten, oder besser gesagt bürgerschaftlichem Engagement im Rahmen der Nahversorgung innerhalb und vor allem ausserhalb der Familien zu. Deshalb müssen alternative Konzepte aufgezeigt werden, die es gerade den betroffenen Bevölkerungsgruppen erlauben, am Konsum und am sozialen Leben zu partizipieren. Das Ehrenamt, das auch als «freiwillige soziale Tätigkeit» oder «bürgerschaftliches Engagement» bezeichnet wird, erlebt derzeit eine grosse Aufmerksamkeit in der öffentlichen Diskussion (vgl. NEU 2011). KüNEMUND \& SCHUPP (2008: 145) sprechen in diesem Zusammenhang auch vom Strukturwandel des Ehrenamts und führen an, dass

«auf der Mikroebene der Strukturwandel des Ehrenamts vor allem als Motivwandel diskutiert und vor dem Hintergrund der Individualisierungs- und Wertewandeldiskussionen plausibilisiert»

wurde. MaI \& Swiaczny (2008: 7) beobachten den Bedeutungszuwachs von individuellen sozialen Tätigkeiten fernab von fest strukturierten oder institutionalisierten Organisationen und sprechen auch von «Individualisierung und Pluralisierung des bürgerschaftlichen Engagements», da dieses positive Wir- kungen auf den Einzelnen sowie die Gesellschaft als Ganzes hervorruft. Vor dem Hintergrund der Nahversorgungsthematik ist das soziale Ehrenamt von grosser Bedeutung. Nichtsdestotrotz zeigen Untersuchungen, dass sich Potential und Anzahl der Engagierten positiv entwickeln. Auch bei den jüngeren Bevölkerungsgruppen zeigt sich eine hohe ehrenamtliche Beteiligung, sodass schon früh Grundsteine für ein bürgerschaftliches Engagement im Alter gelegt werden (MAI \& SWIACZNY 2008).

Um lediglich die Bereitstellung mit Waren zu sichern, kann das Internet mit dem sogenannten $e$-commerce einen Beitrag leisten. Dieser Markt wächst kontinuierlich und darf nicht vernachlässigt werden (vgl. Burt et al. 2010: 188; PurPer 2007: 36), stellt aber für die gesellschaftliche Partizipation der potentiellen Nutzer keinen Mehrwert dar und soll deshalb in diesem Beitrag keine weitere Erwähnung finden. Überdies stellt die mobile Versorgung eine sowohl konsumbefriedigende als auch gesellschaftlich-partizipierende Möglichkeit für die Bevölkerung der ländlichen Regionen dar, wird jedoch oft als Auffanglösung nach dem Wegfall stationärer Versorger angesehen (vgl. HAHne 2009). Dieser Beitrag konzentriert sich auf den Bereich des bürgerschaftlichen Engagements und muss auf eine intensivere Auseinandersetzung mit anderen Aspekten verzichten.

\section{Bevölkerungsdynamik und Nahversorgung im ländlichen Raum Nordhessens}

Die Region Nordhessen umfasst neben der kreisfreien Grossstadt Kassel die Landkreise Kassel, WaldeckFrankenberg, Hersfeld-Rothenburg, Fulda sowie den Schwalm-Eder-Kreis und den Werra-Meissner-Kreis. Der überwiegende Teil der Region ist durch grossflächige Gemeinden mit zahlreichen kleinen Dörfern gekennzeichnet. Ausnahmen bilden der Verdichtungsraum Kassel, welcher aus der Stadt Kassel und wenigen umliegenden Kommunen besteht, das Oberzentrum Fulda sowie einige verdichtete Siedlungsbereiche, die meist die relativ starken Mittelzentren der Region umfassen. Allein in den Jahren 2000 bis 2009 nahm die Einwohnerzahl in der Region um rund drei Prozent ab (Hessisches Statistisches Landesamt 2010). Nach den Prognosen des Regionalplanes Nordhessen werden im Jahr 2020 mehr als 75.000 Menschen weniger in der Region leben als 2005 (REGIONALVERSAMMLUNG NoRDHESSEN 2009).

Allein in der Stadt und im Landkreis Kassel war 2009 rund ein Drittel der über 20.000 Einzelhandelsunternehmen der Region angesiedelt. Diese verteilen sich demnach nicht gleichmässig im Raum, sondern sind meist auf die Ober- und Mittelzentren in der Region 


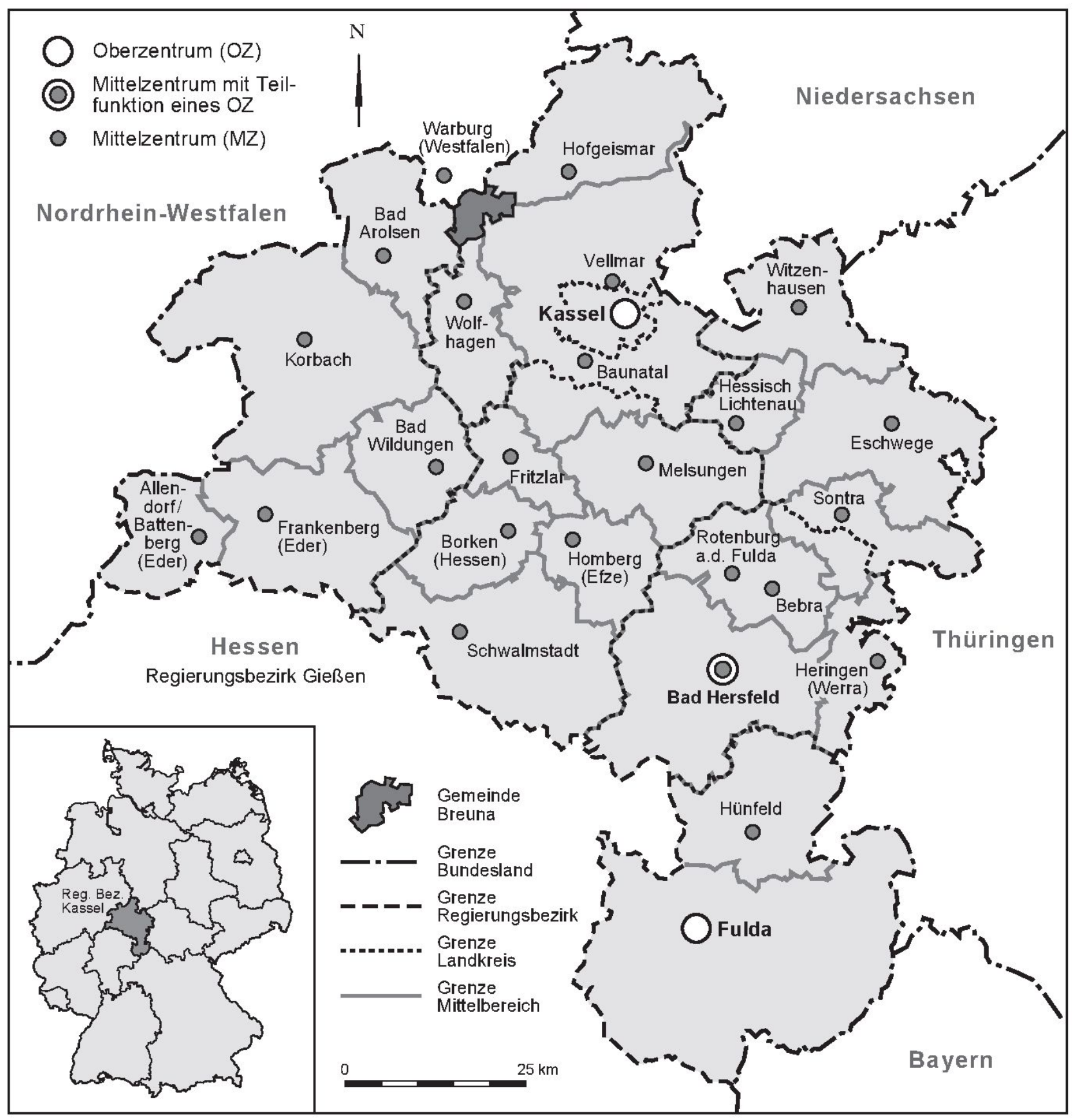

Abb. 1: Zentrale Orte und Mittelbereiche in Nordhessen Central places and catchment areas in northern Hesse Lieux centraux et zones de chalandise en Hesse du Nord Kartengrundlage: RegionalPlan Nordhessen 2009: 31; Kartographie: C. MANN

konzentriert (vgl. Abb. 1). Zur Nahrungsmittelbranche gehören davon in etwa 4.000 Einzelhandelsbetriebe (INDUSTRIE- UND HANDELSKAMMER Fulda 2009; INDUSTRIE- UND HANDELSKAMMER KASSEL 2009). In der Region sind vorrangig vier der grössten deutschen Lebensmitteleinzelhandelsakteure (Edeka-Gruppe,
Rewe-Gruppe, Schwarz-Stiftung mit Lidl und AldiNord) vertreten. Die Metro-Group ist lediglich in den grösseren Zentren der Region anzutreffen. Hinzu kommen einige wenige, teils regionale Unternehmen, wie beispielsweise die Supermarktkette Tegut...Gutberlet Stiftung \& Co. aus Fulda. Durch die Konsolidie- 
rung im Lebensmitteleinzelhandel, also die Herausbildung eines Oligopols (AoYama 2007; BurT et al. 2010), einhergehend mit der Internationalisierung sowie einer stetigen Vergrösserung der Verkaufsflächen, verschwinden die Klein- und Kleinstbetriebe zusehends (DaWson 2010; KulKe 1992; WorTMann 2003).

Während HEINRITZ et al. (2003) die handelsendogenen Einflüsse des sogenannten Strukturwandels auf drei Hauptfaktoren reduzieren, spricht Dawson (2010) ergänzend von einer grösseren Komplexität der Organisationsstrukturen innerhalb der grossen Einzelhandelsunternehmen. Überdies unterstreicht DawsoN (2010) die Relevanz von lokalen Kulturen («local cultures»), welche erheblichen sozialen, ökonomischen, politischen und technologischen Veränderungen unterliegen. Wie können jedoch nun die durch den zweiten demographischen Übergang und den Strukturwandel im Einzelhandel entstehenden und bereits entstandenen Versorgungslücken geschlossen werden, um die Nahversorgung in den ländlichen Regionen zu sichern?

\subsection{Der Dorfladen}

Eine Möglichkeit zur Sicherung der Nahversorgung ist die Einrichtung von Dorfläden auf Basis bürgerschaftlichen Engagements, wobei «der eigentümergeführte Laden immer die bessere Variante ist», so ein Vertreter des Amtes für den ländlichen Raum des Landkreises Kassel. Ein Vertreter des Einzelhandelsverbandes Hessen-Nord steht den inhabergeführten Lebensmittelläden ebenfalls positiv gegenüber, merkt aber auch an, dass manche Standorte mit einem Jahresumsatz von $150.000 €$ lediglich eine «Kümmerexistenz» führen und dass das vorhandene Umsatzpotential in solchen Fällen nicht ausgeschöpft werden kann. Dem ehrenamtlich betriebenen Lebensmittelladen werden keine grossen Chancen eingeräumt, denn es bedarf einer gewissen Kaufkraft, damit solche Dorfläden auch langfristig wirtschaftlich betrieben werden können. Auch von Seiten des Landkreises WaldeckFrankenberg wird angeführt, dass «die Nahversorgung in der Wirtschaft bleiben und professionell betrieben werden muss». Doch hier tritt wiederum ein Problem für die älteren Menschen auf, denn gerade diese benötigen eine wohnortnahe Versorgung, aber «haben vom Bedarf her nicht so viel, dass sich ein Laden tragen würde» (Interview mit Vertreter der Industrie- und Handelskammer Kassel). Das heisst, auch wenn ein Einkommen in ausreichendem Masse vorhanden ist, haben die Senioren ein von den jüngeren Bevölkerungsgruppen vor allem im Umfang differierendes Kaufverhalten. Eine Gefahr für kleine Lebensmittelläden ist jedoch die Tatsache, dass die Kunden lediglich eine kleine Auswahl an Waren vorfinden, denn auf der geringen Verkaufsfläche können nicht alle Sortimente in der Breite und Tiefe angeboten werden. Dies macht das Einkaufen im Dorfladen nicht zu einem Erlebnis, was heutzutage eine grosse Rolle bei den Konsumenten spielt. Des Weiteren ist der Verbraucher immer

«bequemer geworden, das heisst er geht dann nicht noch in den Dorfladen, geht dann noch zum Metzger und dann zum Getränkelieferanten, sondern er bringt sich seine Sachen zentral irgendwo mit» (Interview mit Ortsvorsteher).

Hier zeigt sich, dass der Konzentrationsprozess im Lebensmitteleinzelhandel für den Konsumenten nicht generell von Nachteil ist. Jedoch kommen die Umsätze nicht den Anbietern vor Ort zugute. Voraussetzung für einen gut funktionierenden Dorfladen ist demnach, dass die Betreiber von eben jenem Laden langfristig leben können. Der Verein Region Kassel-Land e.V. hat in einigen Ortschaften Machbarkeitsstudien für die Errichtung von ehrenamtlichen Dorfläden gefördert.

«Da sieht man aber schon, dass selbst in grösseren Dörfern dieses Problem kritisch gesehen wird. [...] Auch die Palette an Angeboten können solche kleinen Läden nicht gewährleisten. Es ist so, dass die Projekte über die Machbarkeitsstudien in den letzten Jahren nicht mehr hinausgegangen sind. [Diese] auch zu dem Ergebnis gekommen sind, es macht wirtschaftlich keinen Sinn. [...] Es liegt eigentlich daran, dass [diese Läden] wahrscheinlich irgendwie zu Wenige nutzen wollten» (Interview mit Vertreterin von Region Kassel-Land e.V.).

Auch im Amt für den ländlichen Raum des Landkreises Kassel wird die Notwendigkeit der wohnortnahen Versorgung mit Lebensmitteln erkannt,

«da dieser Bereich im Rückzug begriffen [ist] und wir ihn nicht halten können. Aber wir kämpfen sehr dagegen an, wir unterstützen Bürgerschaftsläden, wir unterstützen Existenzgründungen im Ladenbereich» (Interview mit Vertreter des Amtes für den ländlichen Raum des Landkreises Kassel).

Das Fehlen einer Versorgungsmöglichkeit wirkt sich negativ auf die Wohn- und Lebensqualität der Bevölkerung aus. Fehlt die wohnortnahe Versorgung, «bedeutet dies auch den Wegzug von Senioren, die sich nicht selber versorgen können» (Interview mit Leiterin des Mehrgenerationenhauses des Landkreises Kassel). Gerade für die ältere Bevölkerung werden die Dorfläden als wichtiger Bestandteil nicht nur der Versorgung, sondern auch des sozialen Zusammenlebens erachtet. Ein wichtiger Aspekt,

«was der Bevölkerung bewusst sein muss [ist], dass man so [einen Dorfladen] nicht nur im Notfall nutzt, sondern regelmässig, um solche Läden überhaupt zu erhalten und damit vor allem älteren Menschen [das Einkaufen] zu ermöglichen» (Interview mit Kreistagsabgeordneter).

Die hier beschriebenen handelsexogenen Faktoren tragen massgeblich zu den Veränderungen der Einzel- 
handelslandschaft bei. Es werden aber auch positive Beispiele genannt, die das Gelingen der Initiierung eines Dorfladens unterstreichen und den Willen einer Dorfgemeinschaft zur Eigeninitiative hervorheben.

«Da gibt es Orte, da haben sich Leute aufgemacht und haben gesagt: Wir wollen einen Bürgerladen haben, den betreiben wir dann auch selbst. Der Lebensmittelladen ist das Zentrum, alles andere kommt dann drum herum. Bank, Post und solche Sachen, das ist auch wichtig, aber der Lebensmittelladen, das ist das, was wirklich zählt im Dorf» (Interview mit Vertreter des Amtes für den ländlichen Raum des Landkreises Kassel).

Jedoch «so richtig gut laufende Dorfläden, die auf ehrenamtlicher Basis funktionieren, sind hier als langfristig erfolgreich nicht bekannt» (Interview mit Vertreter des Einzelhandelsverbandes Hessen-Nord e.V.). «Wir haben einen ehrenamtlich geführten Dorfladen in 190 Orten», führt der Vertreter der Stabsstelle Ländlicher Raum des Landkreises Waldeck-Frankeberg an und unterstreicht damit das bisher geringe Vorkommen dieser Form der Nahversorgung. Nachdem der letzte Lebensmittelladen 2003 aufgrund des Alters der Inhaber sowie fehlender Nachfolge schliessen musste, «wurde das Defizit bei der Versorgung der Menschen in der Kerngemeinde mit Gütern des täglichen Bedarfs» erkannt (Interview mit Vereinsvorsitzendem Dorfladen Bromskirchen e.V.). Der 2007 gegründete Dorfladenverein bekam von der Kommune ein zinsloses Darlehen, um den Umbau zu einem Lebensmittelgeschäft mit angeschlossenem Getränkemarkt zu realisieren. Das Einzugsgebiet des Ladens umfasst die Kerngemeinde mit ca. 1.400 Einwohnern und zwei zur Kommune zugehörige Nachbarorte, wobei vorrangig ältere Menschen und in Teilen jüngere Familien dort regelmässig einkaufen. Auf einer Fläche von $80 \mathrm{~m}^{2}$ wird ein übersichtliches Sortiment an Waren angeboten. Mit einem Umsatz von $290.000 €(2009)$ wird eine geringe Flächenproduktivität von $3.625 €$ erzielt. Nach Abzügen bleibt jedoch ein kleiner Gewinn übrig, aber nur aufgrund der Tatsache, dass die Arbeit von fünf bis sechs ehrenamtlichen Helfern erledigt wird. «Das Ziel ist immer die schwarze Null», betont der Vereinsvorsitzende des Dorfladenvereins und gleichzeitige Bürgermeister der Kommune. Dass nicht in jedem Ort ein Dorfladen existieren kann oder wirtschaftlich überlebensfähig bleibt, ist den Interviewpartnern durchaus bewusst. Doch wird auch thematisiert und betont, dass gerade der Konsument durch ein «bewussteres Einkaufsverhalten» die Entwicklungen im nahen Umfeld dahingehend beeinflussen kann, dass ein kleiner Lebensmittelladen fortbesteht oder neue Konzepte Anklang finden (Interview mit Vertreter der Handwerkskammer Kassel). Es zeigt sich, dass dieses Konzept zwar eine Alternative darstellt, um die Nahversorgung zu sichern, die Bevölkerung muss jedoch derartige Vorhaben aktiv unterstützen.

\subsection{Aktive Nachbarschaftshilfe und weitere Alternativen}

Auf Grundlage der Interviews mit den Akteuren aus der Region konnten verschiedene Ansätze zur Verbesserung der Nahversorgung identifiziert werden, welche auf der Basis bürgerschaftlichen Engagements beruhen. Eine weit verbreitete, aber selten beachtete bzw. kaum in Statistiken erfasste Massnahme ist die aktive Nachbarschaftshilfe. Obwohl diese Form der Unterstützung eher informell stattfindet, gibt es bereits vereinzelt Zusammenschlüsse, «aber das ist noch nicht koordiniert oder vernetzt. Das ist das Ziel der neuen Koordinierungsstelle», berichtet die Bürgerbeauftragte des Landkreises Kassel. Aufgrund der oft engen sozialen Beziehungen zu Menschen im näheren Wohnumfeld besteht gerade für die oft ältere und weniger mobile Bevölkerung ein guter Zugang zu Waren und einfachen Dienstleistungen. So werden die Nachbarn zum Helfer und Nahversorger gleichermassen. Dabei ist das Aufgabenfeld facettenreich und kann das Einkaufen von Lebensmitteln, die Erledigung von Post- und teilweise Bankdienstleistungen, Fahrdienste zu Ärzten, einfache Koch- oder Reinigungsdienste sowie einfach nur Gespräche umfassen. All diese Aktivitäten, die derzeit keiner übergeordneten Organisierung unterstehen, tragen einerseits zur Versorgung der weniger oder nicht mobilen Bevölkerung bei und schaffen auf der anderen Seite stärkere soziale Bindungen. Dies scheint auch Normalität auf dem Dorf zu sein, denn, «was selbstverständlich ist, ist die direkte Nachbarschaftshilfe» (Interview mit Kreistagsabgeordneter). Jedoch sind sich die Interviewpartner nahezu einig darüber, dass die Versorgung mit Waren und Dienstleistungen in der Fläche mithilfe des Ehrenamtes nicht vorstellbar ist, denn «das überfordert auch das Ehrenamt» schlichtweg (Interview mit Kreistagsabgeordneter). So besteht zwar heute eine grössere Flexibilität und Kreativität im Bereich des Ehrenamtes, jedoch ist «der Wunsch, Freizeit für sich alleine zu verbringen» vorhanden (Interview mit Vertreterin des Regierungspräsidiums Kassel), und der «Trend geht dahin, [...] sich punktuell zu engagieren» (Interview mit Leiterin des Mehrgenerationenhauses des Landkreises Kassel). Zudem wird angemerkt, dass sich die Dörfer hochgradig unterscheiden, was die Initiierung oder das Anstossen neuer und kreativer Ideen sowie das Zusammengehörigkeitsgefühl per se betrifft, denn dies hängt sehr stark von Personen, wie Vereinsvorsitzenden, Ortsvorstehern oder Bürgermeistern, ab.

«Es ist die Frage, wie stark der Gemeinsinn ist und da unterscheiden sich die Dörfer unglaublich voneinander. Und [die Dörfer] können auch umkippen, wenn sie einen neuen Ortsbeirat bekommen. [...] Das habe ich mehrfach erlebt in Orten, die zehn oder 15 Jahre inaktiv waren, und mit einem neuen Ortsvorsteher plötzlich in die 〈Puschen kamen. Und es gab ein Wir-Gefühl und eine dörfliche 
Identität, weil Ansätze plötzlich öffentlich vorgestellt und diskutiert worden sind, $[\ldots]$ und nicht parteipolitisch dominant Entscheidungen getroffen wurden» (Interview mit Vertreterin des Regierungspräsidiums Kassel).

Darüber hinaus gibt es gleichwohl auch von Seiten der Kommune initiierte Projekte, die zur Verbesserung der Versorgungsmöglichkeiten beitragen. So werden positive Beispiele aus der Region genannt, bei denen ältere oder körperlich eingeschränkte Menschen mittels organisierter Fahrdienste zur nächsten Einkaufsstätte transportiert werden oder aber via Fahrradkurier Lebensmittel nach Hause geliefert bekommen. Dabei stellt sich jedoch immer die Frage nach der Wirtschaftlichkeit und langfristigen Tragfähigkeit solcher Projekte (Interview mit Vertreterin von Region Kassel-Land e.V.).

«Ob dieses Wir-Gefühl soweit geht, dass ehrenamtliche Arbeit machbar ist in gewerblichen Strukturen, das ist eine der spannendsten Fragen derzeit» (Interview mit Vertreter des Einzelhandelsverbandes Hessen-Nord e.V.)

Die Möglichkeit zur Versorgung der Bevölkerung im ländlichen Raum mithilfe des bürgerschaftlichen Engagements findet derzeit auf der Ebene der aktiven Nachbarschaftshilfe und weniger auf einer professionell organisierten und auf die Gesamtkommune ausgeweiteten Dimension statt. Grenzen sowie Möglichkeiten der dargelegten Optionen, eben diese auf andere ländliche Raumtypen $\mathrm{zu}$ übertragen, werden im Folgenden aufgezeigt und zusammengefasst.

\section{Fazit}

Aufgrund der geschilderten demographischen und ökonomischen Veränderungen, dargelegt an dem Beispiel Nordhessen, ergeben sich neue Herausforderungen für ländliche Räume in ganz Europa. Die aus der Beispielregion abgeleiteten Erkenntnisse können auf andere Regionen übertragbar sein, wenn deren innere Struktur, z.B. Lage der zentralen Orte und Distanzen zwischen diesen, sowie deren Funktionen ähnlich ausgeprägt sind. Der Formatwandel, die Konsolidierung sowie die Konzentration der Einzelhandelsbetriebe auf die Grund-, Mittel- und Oberzentren haben zur Folge, dass grosse Bereiche in ländlichen Räumen unterversorgt sein werden. Dies hat Auswirkungen auf die Wohn- und Lebensqualität in diesen Regionen, welche die Effekte des zweiten demographischen Übergangs deutlicher zu spüren bekommen als beispielsweise Städte. Sowohl die Kommunen, die Bürgerinnen und Bürger als auch die Einzelhändler müssen auf die Veränderungen reagieren und versuchen dies längst, mehr oder weniger erfolgreich. Als Fazit zu ziehen ist, dass die Errichtung ehrenamtlich geführter Dorfläden stark von engagierten Personen und der Unterstützung sei- tens der Gesellschaft abhängig ist. Nach Ansicht der Experten ist der stationäre, mit ökonomischen Motiven betriebene Lebensmittelladen mittel- und langfristig die bessere Variante. Dies zeigt sich daran, dass es bisher im Untersuchungsraum kaum Beispiele für gut funktionierende, ehrenamtlich geführte Dorfläden gibt. Eine Gefahr für solche Projekte stellt das sich verändernde Konsumverhalten der Bevölkerung dar. Zudem sind die Aspekte Kaufkraft und letztendlich der generierte Umsatz der Dorfladenbetreiber bedeutungsvoll. Die diskutierte Alternative leistet zwar einen Beitrag zur Nahversorgung, kann jedoch die privatwirtschaftlich betriebenen Versorgungseinrichtungen in keinem Fall vollständig ersetzen. Schlussendlich muss den Einwohnern bewusst sein oder gemacht werden, dass sie durch ihr Konsumverhalten bei jedem Einkauf über das Fortbestehen oder die Aufgabe eines Nahversorgers entscheiden. Die aktive Nachbarschaftshilfe ist dagegen eine über die Nahversorgung hinausreichende Variante zur Sicherung des «Dorflebens». Diese bietet grosse Chancen und Potentiale, welche noch ausbaufähig sind, damit die weniger mobile Bevölkerung am Konsum und am sozialen Leben partizipieren kann. Es fehlt jedoch weitestgehend die Datengrundlage für eine Koordinierung und Konzeptionalisierung, was als Schwäche dieser Option gesehen werden muss. Die dargestellten Optionen stellen bisher lediglich ergänzende Möglichkeiten dar, um die Bevölkerung in geringem, aber ausreichendem Masse mit Waren und Dienstleistungen zu versorgen. Bürgerschaftliches Engagement im Rahmen der Nahversorgung sollte als Chance verstanden werden, um die entstehenden Versorgungslücken zu schliessen, jedoch nicht als Patentrezept betrachtet werden.

\section{Literatur}

AoYAma, Y. (2007): Oligopoly and the structural paradox of retail TNCs: an assessment of Carrefour and Wal-Mart in Japan. - In: Journal of Economic Geography $7,4: 471-490$.

Born, K.M. (2011): Ländliche Räume in Deutschland. Differenzierungen, Entwicklungspfade und -brüche.In: Geographische Rundschau 63, 2: 4-10.

Burt, S., Sparks, L. \& C. Teller (2010): Retailing in the United Kingdom - a synopsis. - In: European Retail Research 24, 1: 173-194.

Cirkel, M. \& R. Juchelka (2007): Gesundheit und Mobilität im Alter. - In: Public Health Forum 15, 3: 24-26.

Dawson, J. (2010): Global trends. Retail trends in Europe. - In: KraffT, M. \& M.K. Mantrala (Hrsg.): Retailing in the $21^{\text {st }}$ century: current and future trends. - Berlin, Heidelberg: Springer-Verlag.

Hahne, U. (2009): Zukunftskonzepte für schrumpfende ländliche Räume. Von dezentralen und eigenständigen Lösungen zur Aufrechterhaltung der 
Lebensqualität und zur Stabilisierung der Erwerbsgesellschaft. - In: Neues Archiv für Niedersachsen, Zeitschrift für Stadt-, Regional- und Landesentwicklung 1/2009: 2-25.

Heinritz, G., Klein, K.E. \& M. Popp (2003): Geographische Handelsforschung. - Berlin: Borntraeger.

Hessisches Statistisches Landesamt (2010): Statistische Berichte. Bevölkerungsvorgänge in Hessen im Jahr 2008. - Wiesbaden: Hessisches Statistisches Landesamt.

HilligardT, J. (2010): Standortfaktor «Ambulante ärztliche Versorgung»: Debatten und Strategien im Bundesland Hessen. - In: Raumforschung und Raumordnung 68, 3: 219-229.

InDUSTRIE- UND HANDELSKAMmer Fulda (2009): Wirtschaftsdaten Region Fulda 2009. - http:/www.ihkfulda.de/inhalt/att/WDB.pdf 12.08.2010.

Industrie- Und Handelskammer Kassel (2009): Statistik auf den Punkt. Einzelhandel in Nordhessen. http:/www.ihk.kassel.de/solva_docs/einzelhandel_in_ nordhessen_2009.pdf 10.08.2010.

Kertzer, D.I., White, M.J., Bernardi, L. \& G. GabriELLI (2009): Italy's path to very low fertility: the adequacy of economic and second demographic transition theories. - In: European Journal of Population 25, 1: 89-115.

Kuhlicke, C., Petschow, U. \& H. Zorn (2005): Versorgung mit Waren des täglichen Bedarfs im ländlichen Raum. Studie für den Verbraucherzentrale Bundesverband e.V. - Berlin: Institut für ökologische Wirtschaftsforschung $\mathrm{GmbH}$.

Kunlicke, C. \& U. Petschow (2005): Sozialräumliche Disparitäten in Deutschland. Vom schleichenden Ende der Nahversorgung. - In: Ökologisches Wirtschaften 4: 2-10.

KulKe, E. (1992): Structural change and spatial response in the retail sector in Germany. - In: Urban Studies 29, 6: 965-977.

Künemund, H. \& J. Schupp (2008): Konjunktur des Ehrenamts - Diskurse und Empirie. - In: ErLINGHAGEN, M. \& K. HANK (Hrsg.): Produktives Altern und informelle Arbeit in modernen Gesellschaften. Theoretische Perspektiven und empirische Befunde.-Wiesbaden: VS Verlag für Sozialwissenschaften: 145-163.

KÜPPER, P. (2010): Regionale Reaktionen auf den demographischen Wandel in dünn besiedelten, peripheren Räumen: Ergebnisse einer deutschlandweiten Befragung. - In: Raumforschung und Raumordnung 68, 3: 169-180.

Lesthaeghe, R.J. \& L. Neidert (2006): The second demographic transition in the United States: exception or textbook example? - In: Population and Development Review 32, 4: 669-698.

MaI, R. \& F. SWIACZnY (2008): Demographische Entwicklung. Potenziale für bürgerschaftliches Engagement. $-=$ Bericht des Bundesinstituts für Bevölkerungsforschung 126.
Matuschewski, A. (2010): Stabilisierung der Regionalentwicklung durch Rückwanderung? Theoretische Konzeptionalisierung und empirische Umsetzung am Beispiel von Ostdeutschland. - In: Zeitschrift für Wirtschaftsgeographie 54, 1: 81-95.

MAYRING, P. (2002): Einführung in die qualitative Sozialforschung. Eine Anleitung zu qualitativem Denken. - 5. Auflage, Weinheim und Basel: Beltz.

Metzger, C. (2008): Nah(!)versorgung im ländlichen Raum - Herausforderungen der Zukunft. Ergebnisse einer Untersuchung im nördlichen Landkreis DonauRies. - In: Geographische Handelsforschung 23: 10-13. NEU, C. (2011): Daseinsvorsorge und Bürgerpartizipation. - In: Geographische Rundschau 63, 2: 44-49.

Pinilla, V., Ayuda, M.A. \& L.A. SÁez (2008): Rural depopulation and the migration turnaround in Mediterranean Western Europe: a case study of Aragon. In: Journal of Rural and Community Development 3, 1:1-22.

Purper, G. (2007): Die Betriebsformen des Einzelhandels aus Konsumentenperspektive. - Wiesbaden: Deutscher Universitätsverlag.

Regionalversammlung Nordhessen (2009): Regionalplan Nordhessen 2009. - Kassel.

SiEDHofF, M. (2008): Demographischer Wandel - zum Begriff und Wesen eines Megatrends. - In: Dresdner Geographische Beiträge 13: 3-14.

Verband Region Stuttgart (2001): Das Ende der Nahversorgung? Studie zur wohnungsnahen Versorgung. - http://www.weeberundpartner.de/files/401_ Nahversorgung_Bericht.pdf 24.08.2010.

WALFord, N.S. \& S. KureK (2008): A comparative analysis of population ageing in urban and rural areas of England, Wales, and Poland over the last three census intervals. - In: Population, Space and Place 14, 5:365-386.

Wortmann, M. (2003): Strukturwandel und Globalisierung des deutschen Einzelhandels. - Discussion Paper SP III 2003-202 des Wissenschaftszentrums Berlin für Sozialforschung, Berlin.

\section{Zusammenfassung: Konsequenzen des zweiten demo- graphischen Übergangs für den ländlichen Raum. Alternative Konzepte zur Nahversorgung aufgezeigt an der Region Nordhessen}

Sowohl die Entwicklung der Bevölkerungszahl und -zusammensetzung als auch die Konsolidierung im Lebensmitteleinzelhandel haben starken Einfluss auf die ländlichen Räume. Gerade in diesen Gebieten ist es deshalb umso wichtiger, die Nahversorgung sicherzustellen. Ziel dieses Beitrages ist die Darlegung von Nahversorgungsrealitäten in ländlichen Regionen in Westdeutschland anhand der Ergebnisse einer Untersuchung in der Beispielregion Nordhessen. Die Aussagen der Akteure aus den Bereichen Planung, Förderung, Politik und Ehrenamt einerseits sowie die 
betriebswirtschaftliche Seite der Nahversorger stellen die Grundlage für die diskutierten alternativen Möglichkeiten zur Sicherung der Nahversorgung dar. Ob und in inwieweit diese zur langfristigen Aufrechterhaltung der Versorgung mit Waren des täglichen Bedarfes beitragen können, ist die zentrale Frage des vorliegenden Beitrages.

Schlüsselwörter: zweiter demographischer Übergang, ländliche Räume, bürgerschaftliches Engagement, Nahversorgung

\section{Summary: Second demographic transition and its impacts on rural areas. Alternative approaches for local supply using the case study of northern Hesse, Germany}

Both the changes in number and structure of the population and the consolidation of the food retail market have an effect on rural areas. Especially in these regions, securing local supply is a critical issue. This article investigates local supply realities in northern Hesse to illustrate the general supply situation in rural areas in western Germany. The statements of regional and local players from the fields of spatial planning, promotion, politics and citizens' involvement as well as local suppliers are the basis for discussion about alternative strategies for the provision of local supply. The main question followed in this paper is whether the suggested strategies can contribute significantly to the long term supply of daily products.

Keywords: second demographic transition, rural areas, citizens' involvement, local supply

Résumé: Les conséquences de la deuxième transition démographique en milieu rural. Approches alternatives pour l'approvisionnement local en Hesse du Nord, Allemagne

Les transformations liées au nombre et à la structure de la population ainsi que le processus de consolidation du secteur des produits alimentaires ont de profondes conséquences en milieu rural. Dans ces régions en particulier, l'approvisionnement en biens de consomma- tion courante est crucial. Le but de cette contribution est de décrire les réalités des commerces de proximité dans les milieux ruraux de l'ouest de Allemagne en prenant pour exemple la Hesse du Nord. L'article discute les alternatives possibles en matière d'approvisionnement du commerce de proximité en se fondant sur des entretiens réalisés auprès d'acteurs locaux et régionaux de l'aménagement du territoire, de la promotion économique, d'acteurs politiques, de représentants de la société civile et de détaillants locaux. La question centrale de cette contribution est ainsi de savoir comment et jusqu'à quel point ces alternatives peuvent contribuer au maintien à long terme de l'approvisionnement en biens de consommation courante des régions rurales.

Mots-clés: deuxième transition démographique, milieux ruraux, engagement citoyen, commerce de proximité

Dipl.-Geogr. Florian Warburg, Philipps-Universität Marburg, Fachbereich Geographie, Deutschhausstrasse 10, D-35032 Marburg, Deutschland. e-mail:warburg@staff.uni-marburg.de

\section{Manuskripteingang/received/manuscrit reçu le 4.2.2011}

Annahme zum Druck/accepted for publication/accepté pour publication: 24.6 .2011 